\title{
Second Life for Digital Photography: An Exploratory Study
}

\author{
Ming Nie \\ University of Leicester, United Kingdom \\ Paula Roush \\ London South Bank University, United Kingdom \\ Matthew Wheeler \\ PebblePad, United Kingdom
}

\begin{abstract}
Second Life (SL) is an emerging technology in higher education. Academics are showing strong interest in it, but so far have more imaginative ideas about using it than experience of actual practice. This paper reports on a pilot study of using SL with six undergraduate students studying Digital Photography in October 2008 at the London South Bank University. The students took part in a group activity in SL that involved creating and manipulating virtual cubes, taking digital photos of avatars, putting these images onto the cubes and telling a story about them. The researchers used qualitative methods, namely personal interviews and a focus group. The findings illustrate benefits of SL for the subject of Digital Photography, particularly with regard to engaging students with active learning, gaining publicity for students as photographers and extending research into subcultures. The study demonstrates how SL can be used as a virtual space to create collaborative learning opportunities that might not be easy to generate in real life. It also examines the issue of user identity through avatars and its impact on group discussion. The paper showcases an example of SL being used for media and art education and offers insights into how this practice might be expanded and transferred to other disciplines and contexts in higher education.
\end{abstract}

Keywords: Second Life; Digital Photography; 3D Multi User Virtual Environments; Collaborative learning; Avatar identity

\section{Introduction}

Educators have recently become interested in 3-D Multi User Virtual Environments (MUVEs) such as Second Life (SL), a social software application developed in the US by Linden Labs, saying that "It is a free online virtual world imagined and created by its residents." (http://secondlife.com/whatis). SL is a social environment, not a game, and avatars in it can do more or less whatever their owners like, including teaching and learning (Salmon \& Hawkridge, 2009).

SL was the third most popular social software application in the UK in 2009, after Facebook and YouTube, and is by far the most widely used 3-D MUVE for teaching and learning (Warburton, 2009). Hundreds of universities, mostly in the UK and US, have a presence in SL 
(Kirriemuir, 2008). Salmon (2009) predicted that all universities will have a SL presence in five years.

Despite the rising number of educators now extending their presence in $\mathrm{SL}$, pedagogical frameworks to bring about student learning in SL are rare. Research into 3-D MUVEs for HE is still in its early stages and educators wanting to explore creative approaches in SL may find themselves with very limited resources. Most research to date into using SL as an educational technology has been based on small-scale experiments. Nevertheless, these pilot studies have identified several areas in which SL is particularly beneficial for teaching and learning.

SL offers the most powerful object-creation toolset of any 3-D MUVE (Salmon, 2009). It Interesting and effective implementations have been described in several domains, including creating simulations in Medicine and Health (Boulos, Hetherington, \& Wheeler, 2007), developing role-play scenarios for training medical professionals (The PIVOTE project, see http://www.pivote.info), and replicating cultures and societies for exploring gender roles in Archaeology (Edirisingha, Nie, Pluciennik, \& Young, 2009).

This paper reports on a pilot study in which the potential of SL for enabling collaborative learning through student-created objects is examined. Collaborative learning research is deeply rooted in Vygotsky's (1978) socio-constructivism that views knowledge as socially constructed rather than individually achieved. Studies have shown that 3-D MUVEs such as SL are effective in supporting aspects of collaborative learning including building communities of practice (Jarmon \& Sanchez, 2008), especially in a distance learning context (Bronack, Riedl, \& Tashner, 2006; Dickey, 2005), and in facilitating knowledge sharing and integration in groups (Schmeil \& Eppler, 2008). User-created virtual worlds and objects (Antonacci \& Modress, 2008) has become another interesting area in which to examine the potential of 3-D MUVEs for collaborative learning. An example of this was reported by Good, Howland, \& Thackray (2008), a study in which students collaboratively designed learning experiences for other students.

\section{Potential of SL for Media Studies and the Arts}

3-D MUVEs have attracted interest from academics in media studies and the arts who want to investigate the culture of virtual worlds. For example, an anthropological study of SL culture was undertaken by Boellstorff (2008), who wrote at length about participant observation in SL. Adopting critical theory on subcultures, Bakioglu (2008) researched emergent griefer (avatars that cause trouble in virtual worlds) subcultures in SL.

A few researchers have explored the potential of 3-D MUVEs for teaching critical theory in media and arts. Using ethnographic methods, Delwiche (2006) experimented in the 3-D MUVEs Everquest and SL, using these environments as a substitute for fieldwork in teaching new media critical theory to undergraduate students in a communication course. Working within a situated learning theory framework, Delwiche emphasised a view of 3D-MUVEs as social spaces and introduced participant observation, arguing that 3D-MUVEs "are living, breathing textbooks that provide students with first-hand exposure to critical theory and professional practice."(p.161)

Virtual identity, that is, the identity given by users to their avatars in 3-D MUVEs, has also attracted researchers' attention. For academics to use MUVEs such as SL as an effective 
teaching and learning environment for collaborative work, "establishing identity is a key stage in developing trust" as "collaboration depends to a large extent on trust" (Salmon \& Hawkridge, 2009, p.408). Studies of virtual worlds have shown that establishing identity can be potentially more complicated in virtual worlds than in real life because users can easily change their avatars' "race, class, gender, age, socio-economic background, and even species" (Chan, 2007). Cooper, Dibbell, \& Spaight (2007) investigated users' real selves in contrast to their digital representations in virtual worlds and found that people tend to create avatars as ideal forms of their real selves. Junglas, Johnson, Steel, Abraham, \& Loughlin (2007) examined digital representation through avatars and the process of identity formation and trust building in virtual worlds. They predicted that virtual worlds will have a higher percentage of individuals who feel free to explore their identities and are less committed to their role, compared to the real world, because of the higher level of anonymity a virtual world offers. In virtual worlds it is more difficult to tell a person's true identity as he or she can establish identity in a much more flexible way than in real life and there are fewer visual or aural cues.

SL is one of the most photographed 3-D MUVEs, with snapshots of its residents and locations circulating abundantly online and in the media. SL therefore presents a unique teaching and learning environment for a degree programme in Digital Photography. Placed within the debates and research discussed above, this pilot study aimed at examining the following three research questions:

1. What can SL offer to the subject of Digital Photography?

2. What can SL offer to enable collaborative learning?

3. What do participants think of the identities that are established through avatars?

\section{The Pilot Study}

\section{The Context}

The study was carried out by the University of Leicester, within the MOOSE project (http://www.le.ac.uk/moose). MOOSE was funded in 2008 by the UK's Joint Information Systems Committee (JISC), under its Next Generation Technologies and Practice programme. The objective of JISC is to "inspire UK colleges and universities in the innovative use of digital technologies, helping to maintain the UK's position as a global leader in education." (http://www.jisc.ac.uk/aboutus.aspx).

The $[\mathrm{M}]$ project focused on design and experiment with activities in SL to enable groups of students to engage with productive learning. Three case studies were conducted within a postgraduate distance learning course in Archaeology and a postgraduate campus-based course in Media and Communications at the University of Leicester, and a campus-based undergraduate course in Digital Photography at the London South Bank University. This paper reports on the third case study.

\section{The Course}

The course Photographic Index was launched in 2007 in the Arts Media and English Department of the London South Bank University. It was designed to teach visual methodologies to first year digital photography students. The students develop work around 
youth subcultures through offline studies of young Muslim girls in London's East End, London's South Bank skate boarders and BMX riders, and online studies of pop cosmopolitan and fandom communities.

Through art- and photo-based research, students explore critical cultural theory, with a particular focus on studies of youth cultures. They debate the concepts of subcultures, neotribe and life styles. They study earlier subcultural theory as developed by Hebdige (1988) and its sociological explanations of the relationship between social class, street fashion, style and the ambiguous politics of youth as a spectacle. Ethnographic methods, with their focus on the participant observer, the fieldwork approach and the use of photography, supplemented by interviews and personal documents, were introduced as a way to shift the unequal power relations between photographer and subjects.

In this pilot, we wanted to explore whether a 3-D virtual world such as SL provided a teaching and learning environment for research into youth cultures and subcultures. The activities designed for the SL pilot had no link to the course's learning outcomes and student performance in these activities was not assessed.

\section{Participants}

The participants were six students, volunteers, studying the BA in Digital Photography. They were all full time campus-based students in their second year, aged just under 20. Five were female and one was male. Two were British, two from Germany, one from Poland and one from Barbados. They attended three 2-hour sessions in SL over three consecutive weeks, meeting face to face in the media lab, to work synchronously in SL.

\section{Design of SL Activities}

The researchers used the idea of virtual story cubes to work in SL. We designed three activities in SL (SL-tivities), based on Salmon's (2004) 5-stage model and the concept of e-tivities (Salmon, 2002), each focusing on interesting collaborative tasks, thus scaffolding students' learning and group work.

In SL-tivity 1, titled "Snapshot tools in SL", students were shown how to use the snapshot function to take photos in SL. They started by snapping their way around the University of Leicester's Media Zoo island (http://slurl.com/secondlife/Media\%20Zoo/177/222/24/) in SL, and then explored other SL territories, their avatars and subcultures, well known for their vibrant looks and fashion styles. Students were encouraged to use the chat tool to participate in a discussion in which each explorer had to explain what they liked about SL and what they saw as opportunities for further photographic exploration. By the end of this activity, each student was expected to have 6-12 digital images in relation to subcultures.

In SL-tivity 2, titled "Story cubes", students were shown how to create a virtual cube, change its size and texture and move it around. Each student then produced his or her own cube with digital images added, taken from SL-tivity 1 . In this task, a new level of complexity was introduced with the uploading of images onto the faces of story cubes. After the task was completed, students were instructed to participate in a discussion in which they had first to 
explain how they came to create their story cube photo-narrative; then they debated the different narratives generated by each photographer.

In SL-tivity 3, titled "Explorations", the aim was for students to explore further and debate the wider potential of SL for digital media and photography in relation to research into youth cultures and subcultures. In this task, the collaborative story cube was further developed. To create the group's story cube and tell a story about it, the individual cubes had to be arranged together: This required some negotiation about which snapshots to keep and which to hide. After the task was completed, students debated the different narratives that were developed in the group's story cube by working together.

This debate further involved students in a critical discussion in which they reflected on issues related to their training in SL, comparing and contrasting it to previous experiences of researching subcultures in real life. The debate included considering how certain image-making possibilities in SL, such as zooming in without being noticed or flying over something to take photographs, impacted on their photographic practice and ethical relationship with other second lifers.

Snapshot 1 and 2 show student activities around virutal story cubes in SL.

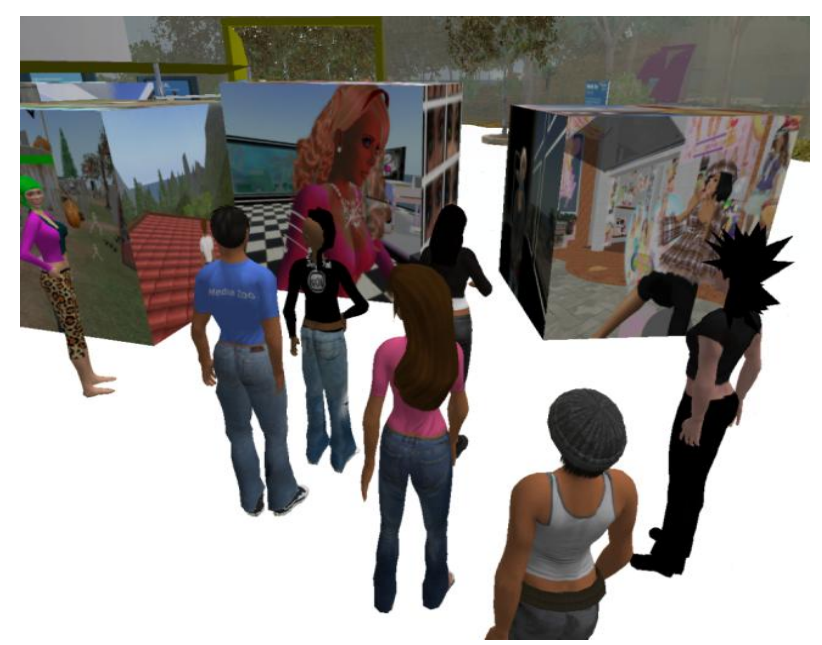

Snapshot 1. The individuals' virtual story cubes. This snapshot illustrates a student avatar explaining to the group about her individual story cube in SL. 


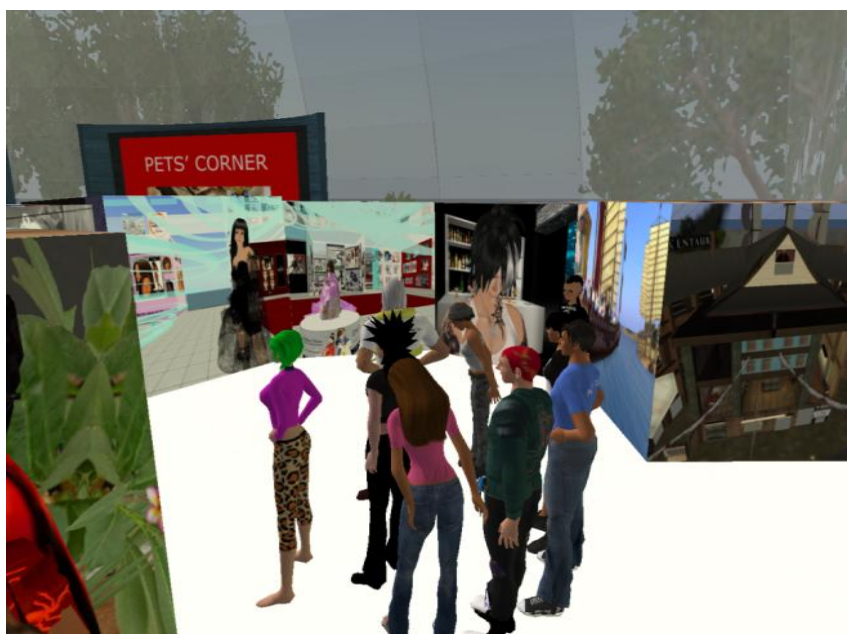

Snapshot 2. The group's virtual story cubes. This snapshot illustrates student avatars developing the group's story cubes collaboratively in SL.

\section{Training in How to Use SL}

The training in how to use SL was conducted in two steps. The first step focused on helping individuals to gain access to $\mathrm{SL}$, creating an avatar and choosing its avatar name, logging on, teleporting, movements and chatting tools. Participants could use a SL guide to learn individually (Wheeler \& Salmon, 2008). It incorporated YouTube videos that demonstrated the basic skills. The second step focused on acquiring competence in more sophisticated in-world skills such as movement, gestures, navigation, camera control, private chat, searching and use of simple development tools. The students acquired these skills through a one-hour face-toface training session together in a media lab.

\section{Research Method}

Student experience with the three SL-tivities was researched using qualitative methods. Each student was interviewed face-to-face and all six took part in a focus group in October 2008, soon after they had completed the three SL sessions. The individual interviews and the focus group lasted for 40-60 minutes each. The interviews captured students' prior experience with $\mathrm{SL}$, their engagement with the three SL-tivities and group work, their perceptions of avatars and technical difficulties or barriers in using SL. The focus group elicited students' views and perceptions on the potential of SL for learning Digital Photography. Chat logs of student discussion during each SL session were also recorded.

Data from interviews was coded using data-driven (inductive) coding (Boyatzis, 1998) and analysed using thematic analysis (Boyatzis, 1998; Joffe \& Yardley, 2004) to identify categories and combine categories into themes. It provided evidence for addressing all three research questions of this pilot study. Chat logs provided examples of how group discussion, mediated through avatars, went in SL, providing evidence for the second and third research questions. 


\section{Results}

\section{SL for Digital Photography}

The graphic interface of SL is particularly appealing for students studying photography because they are sensitive and responsive to visuals and colours. Students appreciated the active learning experience enabled by avatars' movements such as walking, flying, teleporting and interacting with other avatars. They liked the hands-on experience of building, developing and manipulating objects, and exploration of photographic techniques such as cameral control and change of environmental settings. One student said in the interview,

"We are photography students. We're all creative artists, visual people that turn on by things that we see. The visual aspect [of SL] is really nice. Walking about, flying about, and seeing all these things people have been created, really colourful. It's just kind of interesting for the eyes. All the little gadgets you got, taking pictures, being able to teleport from one place to another is really nice... I like the idea of learning through it. I think it's quite fun and quite hands-on." (Student 5)

Having found SL appealing, these students saw SL as a virtual place to display their photographic work, advertising themselves as artists and potentially networking with artists all over the world. They found SL cheaper and less space-constrained in running a virtual exhibition or gallery than in real life. One student concluded,

"It's just a completely different way of advertising yourself... If you were having an exhibition in London, you get real people from that area in London to see your pictures, [whereas in SL] you can have people, you can invite people from all over SL coming from all over the world in real life to see your work. It's really good at networking as well as displaying and exhibiting." (Student 3)

Students also considered SL an alternative virtual environment for conducting research into subcultures. They liked being able to visit so many places and look into different subcultures in SL within a short time: it made their research much easier in SL than in real life. One student pointed out,

"Last year we did a project about youth culture. I think SL could be very interesting in terms of research about youth culture as well. I think you can find the same thing as well [in SL], it's just easier... Also I think it wouldn't be as easy as taking pictures from so many different places in real life because you can go to everywhere you like [in SL], and in real life you can't travel to China... So you see different subcultures within a few minutes. That's a bit difference." (Student 2)

Carrying out fieldwork in SL prompted students to consider broader ethical issues in relation to taking digital images of avatars, as one student reflected in the interview,

"[In real life] I have to speak to the real person, ask for their permission to pose for me, while in SL I don't need to because if I took the photo from far away, they are unaware of this at all." (Student 4) 
This fieldwork experience also encouraged students to consider different methods for conducting research in $\mathrm{SL}$, as one student reflected during the focus group,

"It would be interested in following someone who is really serious about SL, someone who spends a lot of time there, who has an occupation in SL and would follow him for days, photographing and interviewing, that would be my kind of approach. Doing more ethnographic work."

In summary, students identified three ways in which SL engaged their learning Digital Photography differently: enabling active learning, gaining publicity as artists and promoting research into subcultures. Table 1 summarises SL's potential for Digital Photography.

Table 1. Potential of SL for Digital Photography

\begin{tabular}{|l|l|}
\hline Findings & Key points \\
\hline \multirow{2}{*}{$\begin{array}{l}\text { Enabling active } \\
\text { learning }\end{array}$} & $\begin{array}{l}- \text { Avatar movements, i.e., walking, flying, teleporting, interacting } \\
\text { taking digital photos }\end{array}$ \\
& $-\begin{array}{l}\text { Exploring photographic techniques, i.e., cameral control, } \\
\text { changing environmental settings }\end{array}$ \\
\hline \multirow{3}{*}{ Gaining publicity } & - Running virtual exhibitions or galleries \\
& - An virtual place for advertisement \\
\hline $\begin{array}{l}\text { Conducting } \\
\text { research }\end{array}$ & - An virtual place for researching into subcultures \\
& - Exploring different research methods \\
\hline
\end{tabular}

At the beginning of this pilot, these students had a preconception that $S L$ is only a game and that people cannot study seriously in it. After participating in the three SL sessions, all six students began to see the potential of SL for teaching and learning.

\section{Creative Collaborations}

All six participants were impressed by how easy it was to build and develop objects collaboratively in SL. One student stated,

\footnotetext{
"It was so easy to create the cubes and put pictures on it, and even negotiate how we're gonna do that. That was incredibly easy. And that really surprised me. So you can learn something definitely." (Student 1)
}

They also highly appreciated that SL allowed them to do things that would not be so easily done in real life, as one student expressed in the interview,

"It was nice because you could do things [in SL] that you wouldn't be able to do [in real life], like building story cubes with these massive blocks, obviously, you wouldn't have the opportunity to do that [in real life]." (Student 5) 
This finding further reinforced students' view that they could learn seriously within SL.

Participants found that the most gratifying aspect of working in SL was working together on the collaborative task, which involved building virtual story cubes, manipulating them and agreeing on the collective narrative. The characteristics of SL kept everyone involved and engaged with what was going on and kept their attention on developing ideas together. One student commented,

"This is interactive, involving you [in] what's going on. It's not like lecturing something when it's quite easy to just sit back and not really being able to pay that much attention. While that [this activity in SL] keeps you involved because it amuses you and it keeps you involved in what's going on. So overall it's definitely a benefit. ... Everyone is involved and engaged what's going on and you switch on like $100 \%$ motivation. I think it's really important because people lose concentration very quickly. You only can concentrate for 20 minutes of the time. So it's really important to keep people interested and definitely SL does that." (Student 5)

Participants very much enjoyed the group support, discussion and negotiation mediated through developing and putting together their story cubes. Box 1 shows an example in which the six students discussed and negotiated with each other about the order in which they should put their story cubes together.

Box 1. Group discussion and negotiation in SL

[6:38] Avatar 1: have you guys decided on an order yet?

[6:39] Avatar 2: I think Barbie, the submissive woman and the one in the black dress should go together

[6:39] Avatar 3: agreed

[6:40] Avatar trainer: which one first?

[6:40] Avatar 2: yep

[6:40] Avatar 3: yeah

[6:40]Avatar 4: but we can place women next to submissive photo

[6:40] Avatar 5: but the black dress links with the oriental theme...

[6:41] Avatar 6: maybe submission first

[6:41] Avatar 2: then Barbie

[6:41] Avatar 5: so surely Barbie, women sub, black dress, kemono lady, bar man, then ship

[6:41] Avatar 3: ok so how about having C's ship in between the submissive/Barbie and the oriental?

[6:41] Avatar 1: please decide, before I screw it up completely

[6:42] Avatar 3: go with what R said

[6:42] Avatar 1: ok

[6:42] Avatar 6: R's idea sounds good

[6:42] Avatar trainer: no one said this was easy!

[6:42] Avatar trainer: you're doing really well!

[6:42] Avatar trainer: good go for it!

[6:42] Avatar 2: yep

[6:42] Avatar 1: anybody does not agree? 


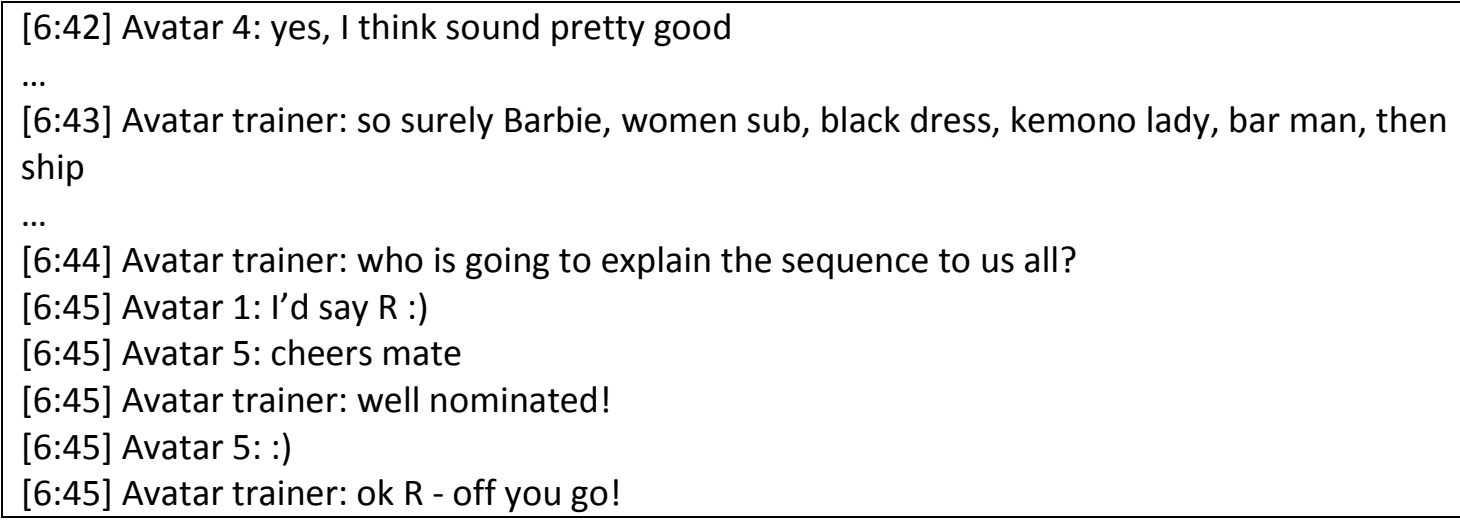

Box 1 shows an example of effective group discussion and negotiation in SL. A key element contributing to this high degree of interactivity and cohesion was that the group discussion was anchored by the development of an interactive object. Students preferred such situated tasks rather than replicating traditional classroom delivery in a virtual environment. One student stated,

"Fieldwork, group work, these are the best approaches for projects in SL. I thought it was kind of weird to have a classroom environment in SL when we are in a classroom environment in a computer anyway. I'd rather be up with my feet in the air in SL. If you can do something different why not do it?" (Focus group)

These students' experiences and reflections on their group task indicate that $\mathrm{SL}$ is a unique environment for creating collaborations. Its characteristics led to easy and productive processes and group work - qualities that are difficult to reproduce and require more effort to generate in real life.

\section{Avatars and Identities}

Communicating with each other through their avatars was a new experience for all the students in this pilot study, but the interviews revealed that they were well aware of the difference between how identity is presented in a virtual world and in real life.

The pilot study showed that students perceived a distinction between in communicating with the real person and interacting mediated through the avatar. They perceived that body language and tones were missing when communicating with each other through avatars. They considered SL an impersonal environment compared to real life, due to these missing personal visual and aural cues. One student said,

"In SL it's not actually me. It's just some digital figures on the screen.... because of this, I just, maybe because I don't have this connection with this person on the screen. They are more like disconnected." (Student 2)

Interestingly, all the students admitted that they behaved in SL the same way as they do in real life. Nobody really took advantage of anonymity in SL and changed or played with their characters. Instead, everyone displayed little inclination to explore and demonstrated a high level of commitment to the group task in SL. Some students made avatars that looked 
completely different from their real selves; the other students, however, were able to recognize them without any difficulty from the way their avatars behaved and interacted in group work.

"You can see little character coming, the dialogues you can see people's humour, and like funny things people are saying, and 'yeah, I know that's them', because you know them in real life." (Student 5)

These findings are not in line with what Junglas et al. (2007) predicated that in virtual worlds, users tend to be more exploratory but lower in commitment than in real life.

The interviews uncovered the causes. The fact that these students knew each other well in real life served as an incentive to their group work in SL. Students had mental pictures of the others when they communicated with each other in SL. They felt that they had talked more to the real person and less to the avatar, as one student commented,

"You kind of got them in your head already how they say something in a text format, and you know how they would be saying anything in real life and their facial expressions to go with that, so you got that inherently in your brain." (Student 5)

As they already knew each other in real life, the relationships had already been built and the trust had already been established. As a result, all the students felt comfortable talking to each other through avatars. All perceived group discussion and negotiation as being fast and efficient in SL despite the fact that their presence was mediated through avatars. They were able to have fun and make jokes exactly the same way as they would in real life or on Facebook. The lack of body language and personal cues seemed to have little impact on their establishment and perceptions of identities in SL. All were able to tell quickly who the person was behind the avatar because they knew each other well in real life.

For the same reason, however, students expressed hesitation in creating their avatars. Observations showed that they created avatars that looked either like themselves or as ordinary as possible. They were worried about being judged by their peers if their avatars looked too weird. As one student pointed out,

"We all know each other and we're sitting next to each other in the same room. There is no room to hide." (Student 1)

This finding challenged a general trend observed by Cooper et al. (2007) that people tend to create avatars in virtual worlds as ideal forms of their real selves.

Additionally, the three sessions in SL were designed and structured almost like teaching sessions, with the tutor giving information and students participating in tasks and discussion and debate. The students felt that they came to these sessions for studying and research instead of having fun with mates. This is another reason why they were reluctant to play with the appearance and characters of their avatars. 


\section{Conclusions and Further Research}

This pilot study shows the potential of SL for the subject of Digital Photography: in engaging students with active learning through avatars' movements and object-creation functionalities, in helping them to gain publicity as artists and in promoting and conducting photo-based field trip and research. It also demonstrates the potential of SL for collaborative learning through learner-created virtual objects, of a kind that could not be easily achieved in real life.

The affordance of SL for enabling collaborative learning within similar subjects or branches in arts has also been exploited by several other UK universities as well. The ATELIER project (http://design.open.ac.uk/atelier-d/) at the Open University used SL to support their design students. Within the Theatron project (http://cms.cch.kcl.ac.uk/theatron/) at the University of Warwick, SL is used to support performing arts students. The findings of our pilot and other projects indicate that if teachers and learners take advantage of social, collaborative and interactive aspects of its virtual environment, SL offers great potential to enable collaborative learning opportunities, especially within subjects in arts, design, media and architecture.

This pilot study also looked into the issue of users' identities created through avatars and its impact on group discussion. As all the participants knew each other well in real life, all perceived the group discussion being fast and efficient in SL despite the fact that their presence was mediated through avatars. For the same reason, however, students expressed hesitation in making their avatars as freely and creatively as they wished, as they were worried about being judged by their peers.

SL offers a rich environment in which digital identities can be explored. The findings from this pilot regarding identities through avatars were generated from a group of campus students who had studied together for almost two years. The results might be different if the study were conducted within a group of distance learners who did not know each other at all. It would be interesting to explore further the issues of virtual identity and the process of trust building amongst distance learners in SL. Among campus students it would be interesting to encourage curiosity about differences, in a space where flexible identity facilitates identity experimentation. For instance, users' identity in SL can be changed through different SL names or appearance. Most importantly, SL can allow and encourage students to experiment with different roles.

\section{References}

Antonacci, D. M., \& Modress, N. (2008). Envisioning the educational possibilities of usercreated virtual worlds. AACE Journal, 16(2), 115-126.

Bakioglu, B. S. (2008). Spectacular subcultures of Second Life: Looking beneath the Lulz. Retrieved from http://palefirer.com/blog/?p=166

Boellstorff, T. (2008). Coming of age in Second Life: An anthropologist explores the virtually human. Princeton, New Jersey: Princeton University Press.

Boulos, M. N., Hetherington, L., \& Wheeler, S. (2007). Second Life: an overview of the potential of 3-D virtual worlds in medical and health education. Health Information and Libraries Journal, 24, 233-245. 
Boyatzis, R. E. (1998). Transforming qualitative information: thematic analysis and code development. London: Sage.

Bronack, S., Riedl, R., \& Tashner, J. (2006). Learning in the zone: A social constructivist framework for distance education in a 3-dimensional virtual world. Interactive Learning Environments, 14(3), 219-232.

Cooper, R., Dibbell, J., \& Spaight, T. (2007). Alter Ego: Avatars and their creators. London: Chris Boot Ltd.

Chan, M. J. (2007). Identity in a virtual world. Retrieved from http://www.cnn.com/2007/TECH/06/07/virtual_identity/index.html

Delwiche, A. (2006). Massively multiplayer online games (MMOs) in the new media classroom. Educational Technology \& Society, 9(3), 160-172.

Dickey, M. D. (2005). Three-dimensional virtual worlds and distance learning: Two case studies of Active Worlds as a medium for distance education. British Journal of Educational Technology, 36(3), 439-451.

Edirisingha, P., Nie, M., Pluciennik, M., \& Young, R. (2009). Socialisation for learning at a distance in a 3-D multi-user virtual environment. British Journal of Educational Technology, 14(3), 458-479.

Good, J., Howland, K., \& Thackray, L. (2008). Problem-based learning spanning real and virtual words: A case study in Second Life. ALT-J, 16(3), 163-172.

Hebdige, D. (1988). Hiding in the light: On images and things. London: Routledge.

Jarmon, L., \& Sanchez, J. (2008). The educators coop experience in Second Life: A model for collaboration. Journal of the Research Centre for Educational Technology, 4(2), 66-82.

Joffe, H., \& Yardley, L. (2004) Content and thematic analysis. In D. F. Marks \& L. Yardley (Eds.), Research Methods for Clinical and health Psychology. London: Sage.

Junglas, I. A., Johnson, N. A., Steel, D. J., Abraham, D. C., \& Loughlin, P. M. (2007). Identity formation, learning styles and trust in virtual worlds. The DATA BASE for Advances in Information Systems, 38(4), 90-96.

Kirriemuir, J. (2008). A spring 2008 'snapshot' of UK higher and further education developments in Second Life. Eduserve Foundation. Retrieved from http://www.scribd.com/doc/7063700/A-Spring-2008-snapshot-of-UK-Higher-andFurther-Education-Developments-in-Second-Life

Salmon, G. (2002). E-tivities: The key to active online learning. London: Routledge Falmer.

Salmon, G. (2004). E-moderating: the key to teaching and learning online (2nd ed.). London: Routledge Falmer.

Salmon, G. (2009). The future for (second) life and learning. British Journal of Educational Technology, 14(3), 526-538.

Salmon, G., \& Hawkridge, D. (2009). Out of this world. British Journal of Educational Technology, 14(3), 401-413.

Schmeil, A., \& Eppler, M. J. (2008). Knowledge sharing and collaborative learning in Second Life: A classification of virtual 3D group interaction scripts. Journal of Universal Computer Science, 14(3), 665-677. 
Vygotsky, L. S. (1978). Mind in society: The development of higher psychological processes. MA: Harvard University Press.

Warburton, S. (2009). Second Life in higher education: Assessing the potential for and the barriers to deploying virtual worlds in learning and teaching. British Journal of Educational Technology, 40(3), 414-426.

Wheeler, M., \& Salmon, G. (2008). Second Life: Guide for learning group participants. Retrieved from http://tinyurl.com/mooseSLguides

Correspondence: Ming Nie, Research Associate in E-learning, Beyond Distance Research Alliance University of Leicester, 103-105 Princess Road East, Leicester LE1 7LG, United Kingdom 